\title{
Damping and the Hartree Ensemble Approximation
}

\author{
Mischa Salléa ${ }^{\mathrm{a}}$,Jan Smit ${ }^{\mathrm{a} *}$ and Jeroen C. Vink ${ }^{\mathrm{a}}$ \\ ${ }^{a}$ Institute for Theoretical Physics, University of Amsterdam \\ Valckenierstraat 65, 1018 XE Amsterdam, the Netherlands
}

We study a Hartree ensemble approximation for real-time dynamics in the toy model of $1+1$ dimensional scalar field theory. Damping behavior seen in numerical simulations is compared with analytical predictions based on perturbation theory in the original (non-Hartree-approximated) model.

\section{Introduction}

Dynamics of quantum fields in real time is a lot more complicated than statics in imaginary time and one has to make approximations before giving the problem to the computer [1]. As an improvement on classical dynamics we are studying the Hartree approximation. The Hartree equations of motion for the $\varphi^{4}$ model in $1+1$ dimensions are given by

$$
\begin{aligned}
\ddot{\varphi}_{x} & =\Delta \varphi_{x}-\left(\mu^{2}+\lambda \varphi_{x}^{2}+3 \lambda C_{x}\right) \varphi_{x}, \\
\ddot{f}_{x}^{\alpha} & =\Delta f_{x}^{\alpha}-\left(\mu^{2}+3 \lambda \varphi_{x}^{2}+3 \lambda C_{x}\right) f_{x}^{\alpha} \\
C_{x} & =\sum_{\alpha}\left[\left(1+2 n_{0}^{\alpha}\right)\left|f_{x}^{\alpha}\right|^{2}\right]
\end{aligned}
$$

where $\varphi_{x}=\left\langle\hat{\varphi}_{x}\right\rangle$ is the mean field and the $f_{x}^{\alpha}$ are a complete set of mode functions used for the parametrisation of the operator field $\hat{\varphi}_{x}$ in the Hartree approximation,

$\hat{\varphi}_{x}=\varphi_{x}+\sum_{\alpha}\left(\hat{a}_{\alpha} f_{x}^{\alpha}+\hat{a}_{\alpha}^{\dagger} f_{x}^{\alpha *}\right)$

The initial conditions are specified by a suitable choice for $\varphi_{x}, \dot{\varphi}_{x}$ and the mode functions, as well as the initial occupation numbers $n_{0}^{\alpha}=\left\langle\hat{a}_{\alpha}^{\dagger} \hat{a}_{\alpha}\right\rangle$.

Hartree-like approximations have been widely used, but when the mean field is homogeneous they do not lead to proper thermalization. This may be ascribed to lack of sufficient scattering in the interaction term involving a spatially constant $C_{x}$. We try to improve on this [2] by writing a homogeneous initial density matrix as a superposi-

*Presented by J. Smit

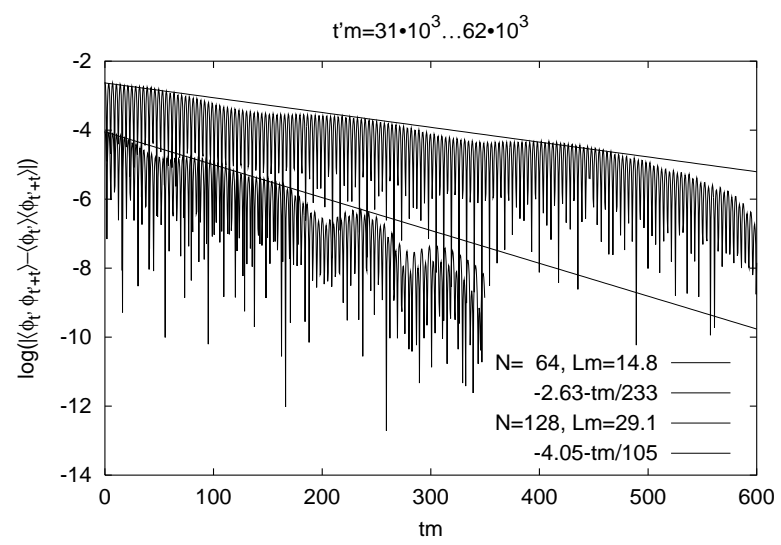

Figure 1. Numerically computed auto-correlation functions $\ln \left|F_{\mathrm{mf}}(t)\right|$ versus time $m t$, with $m$ the temperature dependent mass. The coupling is weak, $\lambda / m^{2}=0.11$ and the temperature $T / m \approx$ 1.4 for the smaller volume (with significant deviations from the Bose-Einstein distribution) and $\approx 1.6$ for the larger volume (reasonably $\mathrm{BE}$ ).

tion of gaussian pure states. The Hartree approximation is then applied to realizations of this ensemble, which are typically inhomogeneous. This allows for scattering of the modes ('particles') via the fluctuating mean field and redistribution over various momenta. To test for thermalization we start out of equilibrium with 'the modes in their vacuum' $\left(n_{0}^{\alpha}=0\right)$ and compute equal-time- and auto-correlation functions. For the resulting particle distributions, see [2]. Here we concentrate on damping phenomena. 
We found funny modulations in autocorrelation functions. Fig. 1 shows such modulations on top of a roughly exponential decay. The correlation function corresponds to the zero momentum mode of the mean field,

$F_{\mathrm{mf}}(t)=\int d x \overline{\varphi(x, t) \varphi(0,0)}_{\text {conn }}$,

where the over-bar denotes a time average, taken after waiting a long time for the system to be in approximate equilibrium. As discussed in [2], this equilibrium is approximately thermal. The function $F_{\mathrm{mf}}(t)$ is analogous to the symmetric correlation function of the quantum field theory:

$F(t)=\int d x\left\langle\frac{1}{2}\{\hat{\varphi}(x, t), \hat{\varphi}(0,0)\}\right\rangle$ conn.

Does $F(t)$ also have the modulations?

\section{Calculation of $F(t)$}

The function $F(t)$ can be expressed in terms of the (zero momentum) spectral function $\rho\left(p^{0}\right)$,

$F(t)=\int_{-\infty}^{\infty} \frac{d p^{0}}{2 \pi} e^{-i p^{0} t}\left(\frac{1}{e^{p^{0} / T}-1}+\frac{1}{2}\right) \rho\left(p^{0}\right)(2)$

which is determined by the retarded selfenergy $\Sigma\left(p^{0}\right)$,

$\rho\left(p^{0}\right)=\frac{-2 \operatorname{Im} \Sigma\left(p^{0}\right)}{\left[m^{2}-p_{0}^{2}+\operatorname{Re} \Sigma\left(p^{0}\right)\right]^{2}+\left[\operatorname{Im} \Sigma\left(p^{0}\right)\right]^{2}}$.

The selfenergy can be calculated in perturbation theory. The relevant diagrams are shown in Fig. 2. The one-loop diagram is present only in

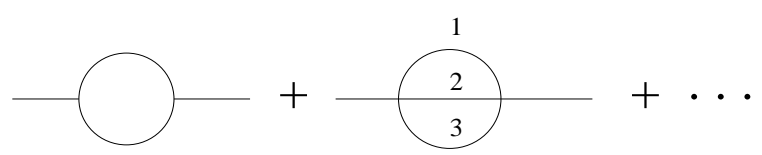

Figure 2. Diagrams leading to thermal damping.

the 'phase of broken symmetry'. It leads to damping for frequencies $p_{0}^{2}>4 m^{2}$, which are irrelevant
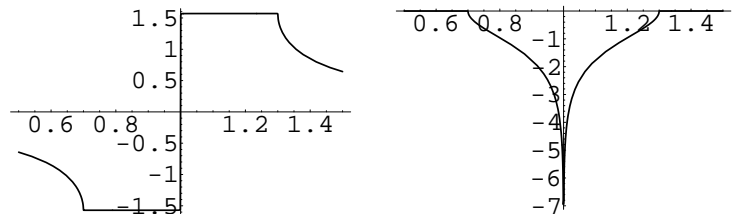

Figure 3. Real (left) and imaginary (right) part of $-i \ln \left(i / z+\sqrt{1-1 / z^{2}}\right)=\arcsin (1 / z)$, for $z=$ $\left(p^{0}+i \epsilon-m\right) /(0.13 m)$ and $\epsilon / m=10^{-4}$.

for the quasiparticle damping at $p_{0}^{2}=m^{2}$. (There is really only a symmetric phase in $1+1$ dimensions, but this is due to symmetry restoration by nonperturbative effects which will not obliterate the damping mechanism described by the diagrams.) So we concentrate on the two-loop diagram. This is given by the sum of two terms, $\Sigma_{1}+\Sigma_{2}$. The first has an imaginary part corresponding to $1 \leftrightarrow 3$ processes, requiring $p_{0}^{2}>9 \mathrm{~m}^{2}$, so it does not contribute to plasmon damping. The second is given by

$$
\begin{aligned}
\Sigma_{2}= & -\frac{9 \lambda^{2}}{16 \pi^{2}} \int \frac{d p_{2} d p_{3}}{E_{1} E_{2} E_{3}} \\
& \frac{\left(1+n_{1}\right) n_{2} n_{3}-n_{1}\left(1+n_{2}\right)\left(1+n_{3}\right)}{p^{0}+i \epsilon+E_{1}-E_{2}-E_{3}} \\
& +\left[\left(p^{0}+i \epsilon\right) \rightarrow-\left(p^{0}+i \epsilon\right)\right],
\end{aligned}
$$

where $\lambda$ is the coupling constant (introduced as $\left.\mathcal{L}_{1}=-\lambda \varphi^{4} / 4\right)$, and

$$
\begin{aligned}
E_{1} & =\sqrt{m^{2}+\left(p_{2}+p_{3}\right)^{2}}, \quad E_{2,3}=\sqrt{m^{2}+p_{2,3}^{2}}, \\
n_{i} & =\left[\exp \left(E_{i} / T\right)-1\right]^{-1}, \quad i=1,2,3 .
\end{aligned}
$$

Its imaginary part corresponds to $2 \leftrightarrow 2$ processes, which contribute to plasmon damping (the regions near $p_{0}= \pm m$ ).

Now the formula for the thermal plasmon damping rate (at zero momentum) in terms of the retarded selfenergy,

$\gamma=-\operatorname{Im} \Sigma(m) / 2 m$,

leads to a logarithmically divergent answer. This is a collinear divergence which is absent in more 


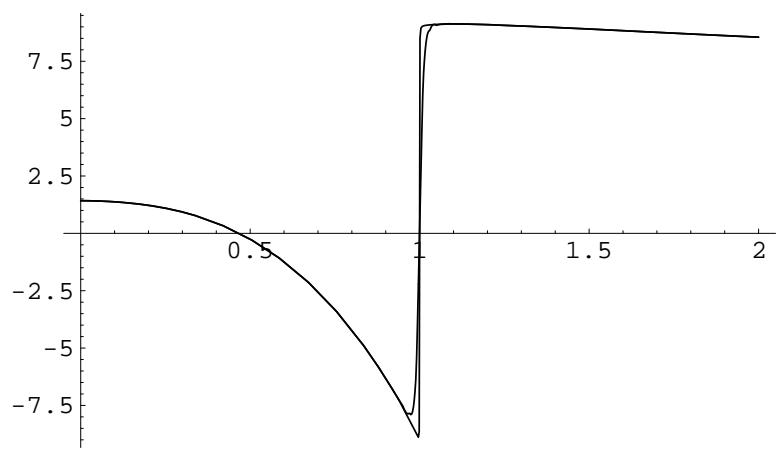

Figure 4. Plot of $\operatorname{Re} \Sigma\left(p^{0}\right) /\left(9 \lambda^{2} / 16 \pi^{2}\right)$ obtained by linear extrapolation $\epsilon=0.02,0.01$ to zero, together with a matching to the logarithmic singularity $(T=m=1)$.

than one space dimension. Inspection shows that the singular part of $\Sigma_{2}$ is given by the nonrelativistic region of the integral in (4). Using polar coordinates $p_{2}=p \cos \phi, p_{3}=p \sin \phi$ this non-relativistic $(p<\kappa \ll m)$ contribution is proportional to

$$
\begin{gathered}
\int_{0}^{\kappa} p d p \int_{0}^{2 \pi} \frac{d \phi}{2 \pi} \frac{1}{p^{0}+i \epsilon-m+\left(p^{2} / 2 m\right) \sin 2 \phi} \\
=m\left[-i \ln \left(\frac{i}{z}+\sqrt{1-\frac{1}{z^{2}}}\right)\right], \\
z=\frac{p^{0}+i \epsilon-m}{\kappa^{2} / 2 m},
\end{gathered}
$$

where $p^{0} \approx m$. This function is plotted in Fig. 3 .

A natural way out of the difficulty posed by the divergent $\Sigma(m)$ may be to continue the selfenergy analytically into the lower half of its second Riemann sheet, $p^{0} \rightarrow m-i \gamma$, and replace (5) by the improved definition

$m^{2}-(m-i \gamma)^{2}+\Sigma(m-i \gamma)=0$.

For weak coupling $\lambda / m^{2} \ll 1$ we then get the equation

$$
\frac{\gamma}{m}=\frac{9 \lambda^{2}}{16 \pi m^{4}} \frac{e^{m / T}}{\left(e^{m / T}-1\right)^{2}}\left[\ln \frac{m}{\gamma}+c(T)\right],
$$

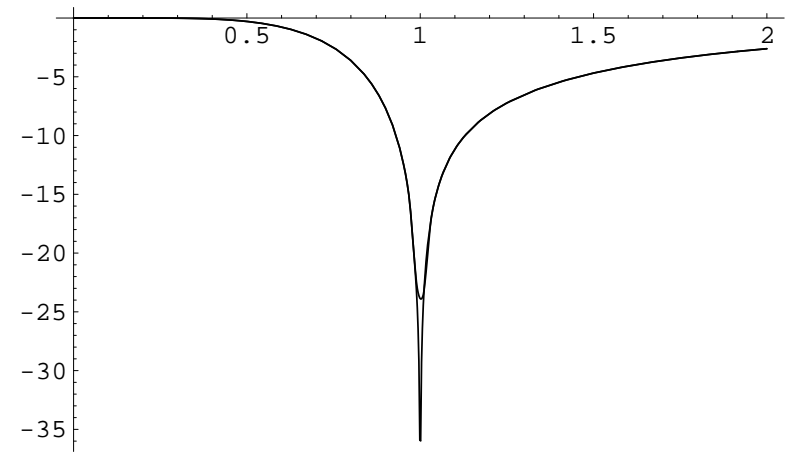

Figure 5. As in Fig. 4 for the imaginary part.

where the constant $c$ has to be determined by matching a numerical evaluation of $\Sigma$ to the form (6) for $p^{0} \approx m$.

We evaluated $\Sigma_{2}$ in (雨) for $T=m$ by numerical integration with $\epsilon / m=0.02,0.01$ and linear extrapolation $\epsilon \rightarrow 0$. The result is shown in Figs. 4 and 5 , together with a matching to the logarithmic singularity, giving $c \approx-0.51$. For example, Eq. (8) now gives $\gamma / m=0.061$, for $\lambda / m^{2}=0.4$.

To see how well this $\gamma$ describes the decay of the correlator $F(t)$ we evaluated this function directly from (2) and (3). The divergence in $\operatorname{Im} \Sigma\left(p^{0}\right)$ at $p^{0}=m$ leads to a zero in the spectral function $\rho\left(p^{0}\right)$. So is there a peak at all in $\rho\left(p^{0}\right)$ ? Fig. 6 shows what happens: the 'usual' peak has separated into two twins!

Fig. 7 shows the resulting $F(t)$. The effect of the double peak is indeed an oscillating modulation on top of the roughly exponential decay. The decay corresponding to $\exp (-\gamma t)$, with $\gamma$ given by (8), is also indicated in the plot: it does not do a good job in describing the average decay beyond the first interference minimum. The 'Twin Peaks' phenomenon implies that the usual definition of damping rate (17) is unreliable in $1+1$ dimensions. Fig. 8 shows the result of a calculation of $F(t)$ with parameters taken from the numerical simulation in Fig. 1 with the larger volume. In this case $\epsilon$ was kept finite, $\epsilon / m=0.005$, which may be more realistic since one expects anyway the 


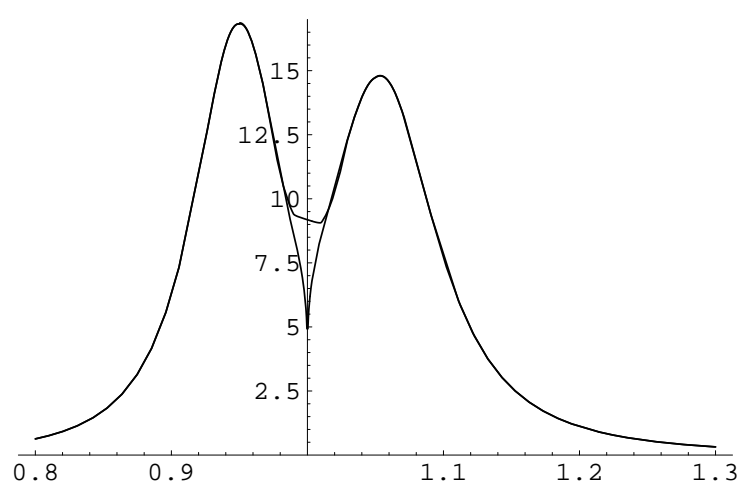

Figure 6. The spectral function $\rho\left(p^{0}\right)$ near $p^{0}=$ $m=1$ corresponding to the selfenergy shown in Figs. $4,5\left(T=m, \lambda=0.4 m^{2}\right)$.

infinity in $\operatorname{Im} \Sigma$ to be smeared out by damping effects in the propagators in Fig. 2. Figs. 1 and 8 are reasonably similar, but how to compare the the average slopes is somewhat ambiguous.

\section{Outlook}

Summarizing, we are encouraged by the similarities in the qualitative features of the numerical and analytical auto-correlation functions. Quantitatively, the damping times are also of the same order of magnitude (105 vs $67 \mathrm{~m}^{-1}$ ), but we did not really accurately compute the relevant autocorrelation function yet in our simulations, which will require a lot more numerical effort.

\section{Acknowledgements}

We thank Gert Aarts for useful conversations. This work is supported by FOM/NWO.

\section{REFERENCES}

1. D. Bödeker, these proceedings.

2. J.C. Vink, these proceedings.

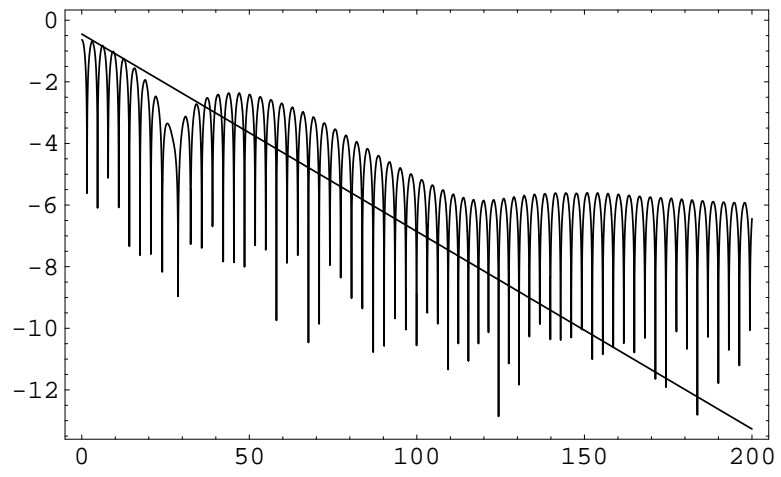

Figure 7. Plot of $\ln |F(t)|$ versus $m t$ for $T=m$, $\lambda=0.4 m^{2}$.

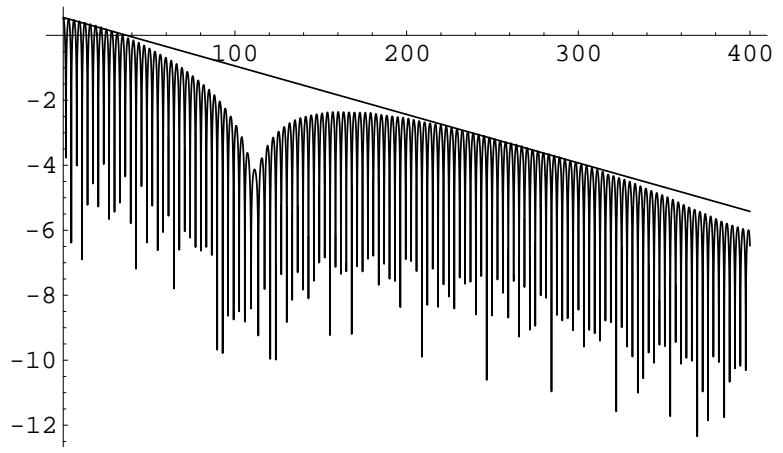

Figure 8. $\ln |F(t)|$ versus $m t$ with $\lambda / m^{2}=0.11$, $T / m=1.63$, corresponding to Fig. 1 . The line is given by $\exp (0.55-t / 67)$. 